\title{
ON THE RATE OF CONVERGENCE OF FINITE DIFFERENCE SCHEMES ON NONUNIFORM GRIDS
}

\author{
FRANK DE HOOG AND DAVID JACKETT ${ }^{1}$
}

(Received 21 June 1983; revised 17 January 1984)

\begin{abstract}
Finite difference schemes for some two point boundary value problems are analysed. It is found that for schemes defined on nonuniform grids, the order of the local truncation error does not fully reflect the rate of convergence of the numerical approximation obtained. Numerical results are presented that indicate that this is also the case for higher dimensional problems.
\end{abstract}

\section{Introduction}

It is well known that finite difference schemes which use uniform spacings are unsatisfactory for problems where the solution has rapid local variation such as boundary layers. The basic problem is that if enough points are used to resolve the layers, the computational effort becomes unacceptably large. On the other hand, if not enough points are used to resolve the layers, the numerical solution may well be a poor approximation even in the interior.

It is therefore necessary to consider finite difference schemes based on irregular grid points. For problems where only first order derivatives need to be discretized, simple finite difference schemes such as the Keller box scheme (Keller [5]) appear to work well and although a general theory is not available, it has been shown by Weiss [7] that for some classes of problems it is necessary only to resolve the layer regions adequately. However it has been found that finite difference schemes with grid spacings that change discontinuously do not yield satisfactory results for second and higher order equations. In fact, Crowder and Dalton [2] claim that even if a large number of points are used in the boundary layers, such schemes can lead to numerical solutions that are worse overall than the numerical

\footnotetext{
${ }^{1}$ Division of Mathematics and Statistics, CSIRO, P. O. Box 1965, Canberra City, A.C.T. 2601.

(c) Copyright Australian Mathematical Society 1985, Serial-fee code 0334-2700/85
} 
solutions obtained using the corresponding finite difference scheme on a regular grid with the same number of points.

The explanation given for this phenomenon (see for example Blottner [1], de Rivas [3], Jones and Thompson [4]) is that the local truncation error may be much larger for a scheme defined on an uneven grid than for the corresponding scheme defined on an even grid. For example (see also Section 2) an elementary Taylor series argument can be used to show that a three point approximation to the second derivative of a function evaluated at a gridpoint can be second order accurate if the grid is uniform but is only first order accurate for an arbitrary grid. Thus, for second order problems, many finite difference schemes used in practice will have a local truncation error that is an order less accurate than the corresponding scheme on a uniform grid. While it is usually possible to devise finite difference schemes on nonuniform grids that are second order accurate, such schemes may have other undesirable properties such as being nonconservative. This fact has motivated the use of smooth coordinate transformations to generate nonuniform grids. For such grids, the local truncation error of a finite difference scheme is of the same order as the local truncation error of the corresponding scheme on a regular grid. Examples of such coordinate transformations may be found in Blottner [1], de Rivas [3], Jones and Thompson [4] and Orszag and Israeli [6].

Although the technique of using coordinate transformations to generate nonuniform grids works well in practice, the justification for its use is misleading in the sense that an assessment of the accuracy of a numerical solution based solely on the order of the local truncation error of the scheme can be incorrect. To support this claim, we examine a class of three point finite difference schemes for the numerical solution of a second order system of ordinary differential equations. It is found that the asymptotic rate of convergence of the approximate solutions obtained by the finite difference schemes with an arbitrary nonuniform grid is the same as the asymptotic rate of convergence of the approximate solutions obtained by finite difference schemes with a nonuniform grid generated by a smooth coordinate transformation. Of course the order of the local truncation error will not be the same. However it is pointed out that it is possible for a scheme to have zero order accuracy for the local truncation error and still be convergent. These analytical results are verified numerically and further calculations are presented that indicate that the results extend to higher dimensions.

\section{Analytical results}

Consider the second order system of ordinary differential equations

$$
\begin{aligned}
(L y)(x) & :=y^{\prime \prime}(x)+P(x) y^{\prime}(x)+Q(x) y(x) \\
& =f(x), \quad 0<x<1,
\end{aligned}
$$


with the boundary conditions

$$
B y:=B_{00} y(0)+B_{01} y^{\prime}(0)+B_{10} y(1)+B_{11} y^{\prime}(1)=b .
$$

Here, $y$ and $f$ are $n$-vector valued functions, $P$ and $Q$ are $n \times n$ matrix valued functions, $B_{r k}, r=0,1, k=0,1$, are $2 n \times n$ matrices and $b$ is a $2 n$-vector. We assume that the problem has a unique solution and, to avoid unnecessary technical detail, that $P, Q$ and $f$ are smooth. Specifically, we assume that $P, Q \in$ $\left(C^{\infty}[0,1]\right)^{n \times n}$ and $f \in\left(C^{\infty}[0,1]\right)^{n}$. Then, $y \in\left(C^{\infty}[0,1]\right)^{n}$ and can be written as

$$
y(x)=\Phi(x) b+\int_{0}^{1} G(x, \zeta) f(\zeta) d \zeta
$$

where $G$ is the Green's function and $\Phi$ is the fundamental solution.

Let

$$
\Delta:=\left\{x_{0}<x_{1}<\cdots<x_{N}\right\}
$$

be a partition and

$$
h_{j}=x_{j+1}-x_{j}, \quad h=\max _{j}\left\{h_{j}\right\} .
$$

For a function $g \in\left(C\left[x_{0}, x_{N}\right]\right)^{n}$ we associate the vector

$$
\hat{\mathrm{g}}_{\Delta}=\left(g\left(x_{0}\right), \ldots, g\left(x_{N}\right)\right)^{T}
$$

and with the vectors $u_{j} \in \mathbf{R}^{n}, j=0, \ldots, N$ we associate the vector

$$
u_{\Delta}=\left(u_{0}, \ldots, u_{N}\right)^{T}
$$

We now discretize the differential equation (2.1a) by

$$
\begin{aligned}
\left(L_{\Delta} y_{\Delta}\right)_{j}:= & 2 R_{j} y_{\Delta}\left[x_{j+1}, x_{j}, x_{j-1}\right] \\
& +S_{j} y_{\Delta}\left[x_{j+1}, x_{j-1}\right]+T_{J} y_{J}=f_{j}, \quad j=1, \ldots, N-1,
\end{aligned}
$$

and the boundary condition (2.1b) by

$$
\begin{aligned}
B_{\Delta} y_{\Delta}:= & \tilde{B}_{00} y_{0}+\tilde{B}_{01} y_{\Delta}\left[x_{0}, x_{2}\right]+2 \tilde{B}_{02} y_{\Delta}\left[x_{0}, x_{1}, x_{2}\right] \\
& +\tilde{B}_{10} y_{N}+\tilde{B}_{11} y_{\Delta}\left[x_{N-2}, x_{N}\right]+2 \tilde{B}_{12} y_{\Delta}\left[x_{N-2}, x_{N-1}, x_{N}\right]=b
\end{aligned}
$$

where

$$
y_{\Delta}\left[x_{j+1}, x_{j}, x_{j-1}\right]=\left(\left(y_{j+1}-y_{j}\right) / h_{j}-\left(y_{j}-y_{j-1}\right) / h_{j-1}\right) /\left(h_{j}+h_{j-1}\right)
$$

and

$$
y_{\Delta}\left[x_{j+1}, x_{J-1}\right]=\left(y_{j+1}-y_{j-1}\right) /\left(h_{j}+h_{J-1}\right)
$$

are the standard divided differences, and $R_{j}, S_{j}$ and $T_{j}=O(1)$.

We shall assume that the scheme $(2.2 \mathrm{a}, \mathrm{b})$ is stable. Specifically, for some $p \geqslant 1$ and constants $C_{1}$ and $C_{2}$ that are independent of $\Delta$,

$$
\left\|y_{\Delta}\right\|_{\infty}<C_{1}\left|B_{\Delta} y_{\Delta}\right|+C_{2}\left\|L_{\Delta} y_{\Delta} s\right\|_{p}
$$


where

$$
\begin{aligned}
\left\|y_{\Delta}\right\|_{\infty} & =\max _{0 \leqslant j \leqslant N}\left|y_{j}\right|, \\
\left\|L_{\Delta} y_{\Delta}\right\|_{p} & =\left(\sum_{j=1}^{N-1} h_{j}\left|\left(L_{\Delta} y_{\Delta}\right)_{j}\right|^{p}\right)^{1 / p},
\end{aligned}
$$

and $|\cdot|$ denotes a fixed finite dimensional norm. Such stability estimates can be established by standard techniques, and the advantage of being able to establish a stability result of (2.3) with $p<\infty$ is obvious. It means for example that if a fixed number of equations in the finite difference scheme (2.2a) are accurate of order zero but the rest are accurate of order one, then the scheme still gives a numerical solution that is asymptotically accurate of order $1 / p$ (provided of course that the boundary conditions ( $2.2 \mathrm{~b}$ ) are consistent). However, in the subsequent analysis we shall take the weakest form of (2.3), namely $p=\infty$. Then,

$$
\left\|L_{\Delta} y_{\Delta}\right\|_{\infty}=\max _{1 \leqslant j \leqslant N-1}\left|\left(L_{\Delta} y_{\Delta}\right)_{j}\right| \text {. }
$$

Since we are primarily interested in the effect of the local truncation error, we will assume that the discretization of the boundary condition is second order. Thus, for any $g \in\left(C^{\infty}[0,1]\right)^{n}$,

$$
B_{\Delta} \hat{g}_{\Delta}-B g=O\left(h^{2}\right) .
$$

We now consider the local truncation error of (2.2a). Using an elementary Taylor series argument, it is easy to verify that for $g \in\left(C^{\infty}[0,1]\right)^{n}$,

$$
2 \hat{g}_{\Delta}\left[x_{j+1}, x_{j}, x_{j-1}\right]=g^{\prime \prime}\left(x_{j}\right)+\left(h_{j}-h_{j-1}\right) g^{\prime \prime \prime}\left(x_{j}\right) / 3+O\left(h^{2}\right)
$$

and

$$
\hat{g}_{\Delta}\left[x_{j+1}, x_{j-1}\right]=g^{\prime}\left(x_{j}\right)+\left(h_{j}-h_{j-1}\right) g^{\prime \prime}\left(x_{j}\right) / 2+O\left(h^{2}\right) .
$$

It is clear from the above that it is still possible to construct schemes for which the local truncation error is second order. The choice

$$
\begin{aligned}
R_{J} & =I-\left(h_{j}-h_{J-1}\right) P\left(\tilde{x}_{j}\right) / 6, \\
S_{J} & =P\left(\tilde{x}_{j}\right)+\left(h_{j}-h_{j-1}\right) Q\left(\tilde{x}_{j}\right) / 3, \\
T_{J} & =Q\left(\tilde{x}_{j}\right), \\
f_{j} & =f\left(\tilde{x}_{j}\right),
\end{aligned}
$$

where

$$
\tilde{x}_{j}=x_{j}+\left(h_{j}-h_{j-1}\right) / 3
$$

for example is second order. However, many schemes that are used in practice will have a local truncation error with the structure

$$
\tau_{j}:=f_{j}-\left(L_{\Delta} \hat{y}_{\Delta}\right)_{j}=\left(h_{j}-h_{j-1}\right) \eta\left(x_{j}\right)+O\left(h^{2}\right)
$$


where $\eta$ is a smooth vector valued function involving derivatives of the solution $y$. The simplest examle of such a scheme is (2.2a) with

$$
\begin{aligned}
& R_{j}=I, \\
& S_{j}=P\left(x_{j}\right), \\
& T_{j}=Q\left(x_{j}\right)
\end{aligned}
$$

and

$$
f_{j}=f\left(x_{j}\right)
$$

Unless some constraint is placed on the partition $\Delta$, the local truncation error $\tau_{\Delta}$ given by (2.5) will be first order. One way to retain a second order truncation error is to use smooth coordinate transformations. If $x_{j}=x\left(\xi_{j}\right), \xi_{j}=j / N$ and $x \in C^{2}[0,1]$, then

$$
h_{j}-h_{j-1}=x\left(\xi_{j+1}\right)-2 x\left(\xi_{j}\right)+x\left(\xi_{j-1}\right)=x^{\prime \prime}\left(\xi_{j}^{*}\right) / N^{2}, \quad \xi_{j+1}<\xi_{j}^{*}<\xi_{j-1} \text {, }
$$

and thus (2.5) will be second order. However, we shall show that stable schemes which satisfy (2.4) and have a local truncation error of the form (2.5) yield numerical solutions that are second order accurate.

Clearly the error

$$
\varepsilon_{\Delta}:=y_{\Delta}-\hat{y}_{\Delta}
$$

satisfies

$$
\begin{aligned}
& \left(L_{\Delta} \varepsilon_{\Delta}\right)_{j}=\tau_{j}, \\
& B_{\Delta} \varepsilon_{\Delta}=b-B_{\Delta} \hat{y}_{\Delta} .
\end{aligned}
$$

Taking the direct analogue of the continuous case we now attempt to approximate $\varepsilon_{\Delta}$ by $\hat{\varepsilon}_{\Delta}$ where

$$
\hat{\varepsilon}(x)=\sum_{k=1}^{N-1} G\left(x, x_{k}\right)\left(h_{k}+h_{k-1}\right) \tau_{k} / 2 .
$$

Using the facts that

(1) $G(x, \xi)$ is smooth for $x<\xi$ and $x>\xi$,

(2) $G(x, \xi)$ satisfies the homogeneous equation

$$
G_{x x}(x, \xi)+P(x) G_{x}(x, \xi)+Q(x) G(x, \xi)=0, \quad \xi \neq x,
$$

(3) $G_{x}(x, x-)-G_{x}(x, x+)=1$, it follows that

$$
\begin{aligned}
\left(L_{\Delta} \hat{\varepsilon}_{\Delta}\right)_{J}= & R, \tau_{j}+\sum_{k \neq j} G_{x}\left(x_{j}, x_{k}\right)\left(S_{j}-R_{j} P\left(x_{j}\right)\right)\left(h_{k}+h_{k-1}\right) \tau_{k} / 2 \\
& +\sum_{k \neq j} G\left(x_{j}, x_{k}\right)\left(T_{j}-R_{j} Q\left(x_{j}\right)\right)\left(h_{k}+h_{k-1}\right) \tau_{k} / 2 \\
& +O\left(h\left\|\tau_{\Delta}\right\|_{\infty}\right)
\end{aligned}
$$


where

$$
G_{x}(x, \zeta)=\frac{\partial}{\partial x} G(x, \zeta), \quad G_{x x}(x, \zeta)=\frac{\partial^{2}}{\partial x^{2}} G(x, \zeta)
$$

In addition, (2.4) implies $\tilde{B}_{00}=B_{00}+O(h), \tilde{B}_{01}=B_{01}+O(h), \tilde{B}_{02}=O(h)$, $\tilde{B}_{10}=B_{10}+O(h), \tilde{B}_{11}=B_{11}+O(h)$ and $\tilde{B}_{12}=O(h)$, and thus

$$
B_{\Delta} \hat{\varepsilon}_{\Delta}=O\left(h\left\|\tau_{\Delta}\right\|_{\infty}\right) \text {. }
$$

Equations (2.6a), (2.6b) lead immediately to the following results.

LEMMA 2.1. Let the local truncation error of the scheme (2.2a) be at least one and let (2.2b) be accurate of order two. Then,

$$
\left\|\varepsilon_{\Delta}-\hat{\varepsilon}_{\Delta}\right\|_{\infty}=O\left(h^{2}\right) .
$$

Proof. Since the scheme is accurate of order one,

$$
\begin{aligned}
& R_{J}=I+O(h), \\
& S_{J}=P\left(x_{j}\right)+O(h),
\end{aligned}
$$

and

$$
T_{j}=Q\left(x_{j}\right)+O(h)
$$

Substitution into $(2.6)$ then yields

$$
\left\|L_{\Delta}\left(\hat{\varepsilon}_{\Delta}-\varepsilon_{\Delta}\right)\right\|_{\infty}=O\left(h^{2}\right),
$$

and

$$
\left|B_{\Delta}\left(\hat{\varepsilon}_{\Delta}-\varepsilon_{\Delta}\right)\right|=O\left(h^{2}\right),
$$

and the result now follows immediately from (2.3).

THEOREM 2.1. Let the local truncation error be of the form

$$
\tau_{j}=\left(h_{j}-h_{j-1}\right) \eta\left(x_{j}\right)+O\left(h^{2}\right)
$$

where $\eta$ is a smooth vector valued function. Then, if (2.4) holds,

$$
\left\|\varepsilon_{\Delta}\right\|_{\infty}=O\left(h^{2}\right) \text {. }
$$

Proof. From Lemma 2.1,

$$
\left\|\varepsilon_{\Delta}\right\|_{\infty}=\left\|\hat{\varepsilon}_{\Delta}\right\|_{\infty}+O\left(h^{2}\right)
$$


Applying summation by parts to $\hat{\boldsymbol{\varepsilon}}_{j}$,

$$
\begin{aligned}
\hat{\varepsilon}_{j} & =\sum_{k=1}^{N-1} G\left(x_{j}, x_{k}\right)\left(h_{k}+h_{k-1}\right) \tau_{k} / 2 \\
& =\sum_{k=1}^{N-1} G\left(x_{j}, x_{k}\right)\left(h_{k}^{2}-h_{k-1}^{2}\right) \eta\left(x_{k}\right) / 2+O\left(h^{2}\right) \\
& =\sum_{k=1}^{N-1}\left\{G_{\zeta}\left(x_{j}, x_{k}\right) \eta\left(x_{k}\right)+G\left(x_{j}, x_{k}\right) \eta\left(x_{k}\right)\right\} h_{k}^{3} / 2+O\left(h^{2}\right) \\
& =O\left(h^{2}\right)
\end{aligned}
$$

where

$$
G_{\zeta}(x, \zeta)=\frac{\partial}{\partial \zeta} G(x, \zeta)
$$

Theorem 2.1, shows that a numerical scheme may yield an approximate solution that is second order accurate even though the local truncation error is only first order. It is also possible to construct numerical schemes that yield convergent numerical solutions but have a local truncation error of order zero. Consider for example (2.2a) with

$$
\begin{aligned}
R_{j} & =I, \\
S_{j} & =P\left(x_{j}\right), \\
T_{j} & =Q\left(x_{j}\right), \\
f_{j} & =\left(1+(-1)^{j}\right) f\left(x_{j}\right),
\end{aligned}
$$

and

$$
h_{j}=h \text {. }
$$

Clearly, $\tau_{j}=(-1)^{j} f\left(x_{j}\right)+O(h)$ and from (2.6) we obtain

$$
\begin{aligned}
& \left\|L_{\Delta}\left(\hat{\varepsilon}_{\Delta}-\varepsilon_{\Delta}\right)\right\|_{\infty}=O(h), \\
& \left\|B_{\Delta}\left(\hat{\varepsilon}_{\Delta}-\varepsilon_{\Delta}\right)\right\|_{\infty}=O(h) .
\end{aligned}
$$

Thus, from (2.3)

$$
\left\|\hat{\varepsilon}_{\Delta}-\varepsilon_{\Delta}\right\|_{\infty}=O(h)
$$

, and since (using summation by parts)

$$
\hat{\varepsilon}_{j}=\sum_{k=1}^{N-1} G\left(x_{j}, x_{k}\right)(-1)^{k}\left(h_{k}+h_{k-1}\right) f\left(x_{k}\right) / 2=O(h)
$$

we have

$$
\varepsilon_{j}=\hat{\mathbf{e}}_{j}+O(h)=O(h)
$$


If we retain higher order terms in (2.6a) and proceed as above it is straightforward though rather tedious to show that

$$
\frac{y_{J+1}-y_{J}}{h_{j}}=y\left(x_{j+1 / 2}\right)+O\left(h^{2}\right)
$$

and

$$
\frac{y_{j+1}-y_{j-1}}{\left(h_{j-1}+h_{j}\right)}=y\left(\frac{x_{j+1}+x_{j-1}}{2}\right)+O\left(h^{2}\right)
$$

for schemes with a local truncation error of the form (2.5).

\section{Numerical results}

To illustrate the theory in the previous section we examine the problem

$$
\begin{aligned}
& y^{\prime \prime}+y^{\prime}-2 y=0, \\
& y(0)=2, \quad y(1)=e+e^{-2} .
\end{aligned}
$$

It is easily verified that the solution is

$$
y(x)=e^{x}+\dot{e}^{-2 x} .
$$

We have applied to this problem the scheme

$$
\begin{aligned}
& 2 y_{\Delta}\left[x_{j+1}, x_{j}, x_{j-1}\right]+y_{\Delta}\left[x_{j+1}, x_{j-1}\right]-2 y_{j}=0, \\
& \left(y_{0}+y_{1}\right) / 2=2, \quad\left(y_{N-1}+y_{N}\right) / 2=e+e^{-2},
\end{aligned}
$$

with

$$
x_{0}=-h_{0} / 2, \quad x_{N}=1+h_{N-1} / 2 .
$$

Two different grid spacings have been used in the computation. The first grid spacing is equally spaced with $h_{j}=h=1 /(N-1)$. For the unequally spaced points, we have taken $h_{2 j}=2 h_{2 j+1}=$ constant. The numerical results are tabulated in Table 3.1 and clearly demonstrate the second order convergence predicted by Theorem 2.1 .

TABLE 3.1

\begin{tabular}{|c|c|c|cc|}
\hline & Uniform grid & & \multicolumn{2}{|c|}{ Nonuniform grid } \\
\hline$N$ & $\max _{1<j<n}\left|y_{j}-y\left(x_{j}\right)\right|$ & ratio & $\max _{1<j<N}\left|y_{J}-y\left(x_{j}\right)\right|$ & ratio \\
\hline 19 & $.1497 \times 10^{-2}$ & 3.915 & $.2632 \times 10^{-2}$ & 3.892 \\
39 & $.3824 \times 10^{-3}$ & 3.957 & $.6762 \times 10^{-3}$ & 3.947 \\
79 & $.9663 \times 10^{-4}$ & 3.978 & $.1713 \times 10^{-3}$ & 3.973 \\
159 & $.2429 \times 10^{-4}$ & & $.4312 \times 10^{-4}$ & \\
\hline
\end{tabular}


In order to illustrate that the previous results possibly extend to higher dimensions, we have also considered the problem

$$
\frac{\partial^{2} u}{\partial x^{2}}+\frac{\partial^{2} u}{\partial y^{2}}=f(x, y), \quad 0<x, y<1,
$$

with the Dirichlet boundary conditions

$$
\begin{array}{lll}
u(x, 0)=g_{1}(x), & u(x, 1)=g_{2}(x), & 0<x<1, \\
u(0, y)=g_{3}(y), & u(1, y)=g_{4}(y), & 0<y<1 .
\end{array}
$$

Specifically, we have considered the problem for which the solution is

$$
u=\exp (x-y) \text {. }
$$

We have applied to this problem the five point scheme

$$
\begin{gathered}
2\left(\left(u_{t+1, j}-u_{i, j}\right) / h_{i}-\left(u_{i, j}-u_{t-1, j}\right) / h_{t-1}\right) /\left(h_{i}+h_{t-1}\right) \\
+2\left(\left(u_{i, j+1}-u_{i, j}\right) / k_{j}-\left(u_{i, j}-u_{i, j-1}\right) / k_{j-1}\right) /\left(k_{i}+k_{t-1}\right) \\
=f\left(x_{i}, y_{j}\right), \quad 0<i, j<N, \\
u_{i 0}=g_{1}\left(x_{t}\right), \quad u_{i N}=g_{2}\left(x_{i}\right), \quad 0<i<N, \\
u_{0 j}=g_{3}\left(y_{j}\right), \quad u_{N j}=g_{4}\left(y_{j}\right), \quad 0<j<N,
\end{gathered}
$$

where

$$
\begin{aligned}
& x_{0}=y_{0}=0, \quad x_{N}=y_{N}=1, \\
& x_{i}=x_{i-1}+h_{i-1}, \quad y_{j}=y_{j-1}+k_{j-1}, \quad 0<i, j<N .
\end{aligned}
$$

Again we have used two grid spacings in our computations. The first are equally spaced points with $h_{i}=k_{j}=1 / N$ while the second grid spacings are nonuniform with $h_{2 \imath}=2 h_{2 \imath-1}=4 / 3 N, k_{2 j}=2 k_{2 j-1}=4 / 3 N$. The numerical results tabulated in Table 3.2 indicate that the convergence in both cases is approximately second order.

TABLE 3.2

\begin{tabular}{|c|c|c|cc|}
\hline & Uniform grid & & \multicolumn{2}{|c|}{ Nonuniform grid } \\
\hline$N$ & $\max _{i, j}\left|u_{i}-u\left(x_{1}, y_{j}\right)\right|$ & ratio & $\max _{i, j}\left|\mathrm{u}_{\mathrm{i},}-\mathrm{u}\left(\mathrm{x}_{\mathrm{i}}, \mathrm{y}_{\mathrm{j}}\right)\right|$ & ratio \\
\hline 20 & $.3289 \times 10^{-4}$ & 3.976 & $.5934 \times 10^{-4}$ & 4.610 \\
40 & $.8272 \times 10^{-5}$ & 3.998 & $.1287 \times 10^{-4}$ & 4.313 \\
80 & $.2069 \times 10^{-5}$ & 3.996 & $.2985 \times 10^{-5}$ & 4.158 \\
160 & $.5173 \times 10^{-6}$ & & $.7178 \times 10^{-6}$ & \\
\hline
\end{tabular}




\section{References}

[1] F. G. Blottner, "Nonuniform grid method for turbulent boundary layers", Proc. 4th Int. Conf. Numerical Methods in Fluid Dynamics, Lecture Notes in Physics 35 (Springer-Verlag, 1975), 91-97.

[2] H. J. Crowder and C. Dalton, "Errors in the use of nonuniform mesh systems", J. Comput. Phys. 7 (1971), 32-45.

[3] K. E. de Rivas, "On the use of nonuniform grids in finite difference equations", $J$. Comput. Phys. 10 (1972), 202-210.

[4] I. P. Jones and C. P. Thompson, "On the use of nonuniform grids in finite difference calculations", Harwell Tech. Rep. A.E.R.E-R 9765 (H.M.S.O., 1980).

[5] H. B. Keller, "Some computational problems in boundary-layer flows", Proc. 4th Int. Conf. Numerical Methods in Fluid Dynamics, Lecture Notes in Physics 35 (Springer-Verlag, 1975), 1-21.

[6] S. A. Orszag and M. Israeli, "Numerical simulation of viscous incompressible flows", Ann. Rev. Fluid Mech. 6 (1974), 281-318.

[7] R. Weiss, "An analysis of the box and trapezoidal schemes for linear singularly perturbed boundary value problems", SIAMJ. Numer. Anal. (to appear). 